\title{
Relevance in Structured Argumentation
}

\author{
AnneMarie Borg and Christian Straßer, \\ Ruhr-University Bochum, Germany \\ annemarie.borg@rub.de, christian.strasser@rub.de
}

\begin{abstract}
We study properties related to relevance in nonmonotonic consequence relations obtained by systems of structured argumentation. Relevance desiderata concern the robustness of a consequence relation under the addition of irrelevant information. For an account of what (ir)relevance amounts to we use syntactic and semantic considerations. Syntactic criteria have been proposed in the domain of relevance logic and were recently used in argumentation theory under the names of noninterference and crash-resistance. The basic idea is that the conclusions of a given argumentative theory should be robust under adding information that shares no propositional variables with the original database. Some semantic relevance criteria are known from non-monotonic logic. For instance, cautious monotony states that if we obtain certain conclusions from an argumentation theory, we may expect to still obtain the same conclusions if we add some of them to the given database. In this paper we investigate properties of structured argumentation systems that warrant relevance desiderata.
\end{abstract}

\section{Introduction}

In this paper we investigate conditions under which the nonmonotonic consequence relation of a given structured argumentation system is robust when irrelevant information is added or removed. Relevance can hereby be understood in two ways. First, syntactically as information that shares propositional variables with the information at hand. Second, semantically, as information that for some reason should not be considered to have defeating power over previously accepted arguments.

Structured argumentation has been studied in various settings such as ASPIC [Prakken, 2010], ABA [Bondarenko et al., 1997], and logic-based argumentation [Arieli and Straßer, 2015; Besnard and Hunter, 2014]. These frameworks share the underlying idea that arguments are to have a logical structure and attacks between them are at least partially determined by logical considerations. Although investigations into translations between these frameworks have been intensified recently [Heyninck and Straßer, 2016], the frameworks are in various aspects difficult to compare and results obtained in one do not easily transfer to others. For this reason, we decided in this paper to study relevance-related properties for structured argumentation on the basis of a simple framework for structured argumentation that allows us, on the one hand, to abstract away from particularities of the systems from the literature and, on the other hand, to translate these frameworks easily. The framework is simple in that arguments are premise-conclusion pairs $(\Gamma, \gamma)$ obtained from a given consequence relation and it only allows for one type of attack (attacks in premises). The obtained simplicity makes studying meta-theory technically straight-forward and the availability of the translations makes results easily transferable.

The paper is structured as follows. In Section 2 we introduce our general setting for structured argumentation. In Section 3 we define the basic relevance-related properties that we will investigate in this paper. In Section 4 we show how many of the most common systems of structured argumentation can be represented in our setting. In Section 5 we prove our main results. We conclude in Section 6.

\section{General Setting}

In the following we work with a simple setting for structured argumentation. It is abstract in the sense that it allows for instantiations that are adequate representations of many of the available systems of structured argumentation such as logicbased argumentation, ASPIC, ABA, etc. (see Sec. 4). In this contribution we restrict ourselves to non-prioritized settings.

We suppose to have available a formal language $\mathcal{L}$ (we denote the set of well-formed formulas over $\mathcal{L}$ also by $\mathcal{L}$ ) and a relation $\vdash \subseteq \wp_{\text {fin }}(\mathcal{L}) \times \mathcal{L}$ (where $\wp_{\text {fin }}$ denotes the set of finite subsets) which we will refer to as the deducability relation. We do not suppose any of the usual Tarskian properties in what follows (reflexivity, transitivity, and monotonicity).

Definition $1\left(\operatorname{Arg}_{\vdash}(\cdot)\right)$. Given a set of formulas $\mathcal{S} \subseteq \mathcal{L}$ we denote by $\operatorname{Arg}_{\vdash}(\mathcal{S})$ the set of $\mathcal{S}$-based arguments: $(\Gamma, \gamma) \in$ $\operatorname{Arg}_{\vdash}(\mathcal{S})$ iff $\Gamma \vdash \gamma$ for $\Gamma \subseteq \mathcal{S}$. Given $a=(\Gamma, \gamma) \in \operatorname{Arg}_{\vdash}(\mathcal{S})$, $\operatorname{Conc}(a)=\gamma$ and $\operatorname{Supp}(a)=\Gamma$.

To accommodate argumentative attacks we suppose to have two functions: a contrariness function ${ }^{-}: \mathcal{L} \rightarrow \wp(\mathcal{L})$ that associates each formula with a set of conflicting formulas and a function $\widehat{\cdot}: \wp_{\text {fin }}(\mathcal{L}) \backslash \emptyset \rightarrow \wp_{\text {fin }}(\mathcal{L})$ that associates support sets with sets of formulas in which they can be attacked. 
Remark 1 . Often $\widehat{\cdot}$ will simply be the identity function, although another option is, e.g., $\widehat{\Gamma}=\left\{\bigwedge \Gamma^{\prime} \mid \emptyset \neq \Gamma^{\prime} \subseteq \Gamma\right\}$.

Definition $2\left(\mathcal{A} \mathcal{F}_{\vdash}\right)$. An (argumentation) setting is a triple $\mathcal{A} \mathcal{F}_{\vdash}=\left(\vdash,{ }^{-}, \widehat{)}\right)$. A setting based on $\mathcal{S} \subseteq \mathcal{L}$ is given by the quadruple $\mathcal{A F}_{\vdash}(\mathcal{S})=(\mathcal{S}, \vdash,-, \cdot)$.

Example 1. A simple example of a setting is $\mathcal{A F}_{\vdash \mathrm{FL}}^{\text {pdef }}=$ $\left(\vdash_{\mathrm{CL}},{ }^{-}\right.$, id) where $\vdash_{\mathrm{CL}}$ is the deducability relation of classical propositional logic and $\bar{\phi}=\{\neg \phi\}$.

Example 2. Another example is the setting $\mathcal{A F}_{F_{\mathrm{CL}}}^{\mathrm{def}}=\left(\vdash_{\mathrm{CL}}\right.$ $, \cdot, \cdot)$ where $\bar{\phi}=\{\neg \phi\}$ and $\widehat{\Gamma}=\{\bigwedge \Delta \mid \emptyset \neq \Delta \subseteq \Gamma\}$.

Definition 3 (Attacks). Given an setting $\mathcal{A} \mathcal{F}_{\vdash}(\mathcal{S})$, where $a=$ $(\Gamma, \gamma) \in \operatorname{Arg}_{\vdash}(\mathcal{S})$ and $b=\left(\Gamma^{\prime}, \gamma^{\prime}\right) \in \operatorname{Arg}_{\vdash}(\mathcal{S})$, a attacks $b$ (in $\phi$ ) iff there is a $\phi \in \widehat{\Gamma^{\prime}}$ for which $\gamma \in \bar{\phi}$.

Our attack form is sometimes called premiseattack [Prakken, 2010] or directed undercut [Besnard and Hunter, 2014]. In Section 4 we will show that by adjusting $\cdot$ and $\hat{\cdot}$ adequately we are able to accommodate many other attack forms defined in the literature.

Definition 4 (Attack Diagram). Given a setting $\mathcal{A F} \mathcal{F}_{\vdash}(\mathcal{S})$, its attack diagram is the directed graph with the set of nodes $\operatorname{Arg}_{\vdash}(\mathcal{S})$ and edges between $a$ and $b$ iff $a$ attacks $b$.

Definition 5 (Dung Semantics, [Dung, 1995]). Where $\mathcal{A} \mathcal{F}_{\vdash}(\mathcal{S})$ is a setting and $\mathcal{A} \subseteq \operatorname{Arg}_{\vdash}(\mathcal{S})$ we define: $\mathcal{A}$ is conflict-free iff there are no $a, b \in \mathcal{A}$ such that $a$ attacks $b$. $\mathcal{A}$ defends $a \in \operatorname{Arg}_{\vdash}(\mathcal{S})$ iff for each attacker $b \in \operatorname{Arg}_{\vdash}(\mathcal{S})$ of $a$ there is a $c \in \mathcal{A}$ that attacks $b . \mathcal{A}$ is admissible iff it is conflict-free and it defends every $a \in \mathcal{A}$. $\mathcal{A}$ is complete iff it is admissible and it contains every $a \in \operatorname{Arg}_{\vdash}(\mathcal{S})$ it defends. $\mathcal{A}$ is preferred iff it is $\subseteq$-maximal complete. $\mathcal{A}$ is grounded iff it is $\subseteq$-minimal complete. $\mathcal{A}$ is stable iff it is admissible and for all $a \in \operatorname{Arg}_{\vdash}(\mathcal{S}) \backslash \mathcal{A}$ there is a $b \in \mathcal{A}$ that attacks $a$.

We denote the set of all admissible [complete, preferred, stable] sets $\mathcal{A}$ (also called "extensions") by $\operatorname{Adm}\left(\mathcal{A F}_{\vdash}(\mathcal{S})\right)$ $\left[\operatorname{Cmp}\left(\mathcal{A F}_{\vdash}(\mathcal{S})\right), \operatorname{Prf}\left(\mathcal{A F} \mathcal{F}_{\vdash}(\mathcal{S})\right), \operatorname{Stb}\left(\mathcal{A F} \mathcal{F}_{\vdash}(\mathcal{S})\right)\right]$ and the grounded set by $\operatorname{Grd}\left(\mathcal{A} \mathcal{F}_{\vdash}(\mathcal{S})\right)$.

Definition 6 (Consequence Relations). Where Sem $\in$ $\left\{\right.$ Grd, Prf, Stb\}, and given a setting $\mathcal{A} \mathcal{F}_{\vdash}$ we define: $\mathcal{S} \sim \sim_{\text {Sem }}^{\mathcal{A} \mathcal{F}_{\vdash}} \phi$ iff for all $\mathcal{A} \in \operatorname{Sem}\left(\mathcal{A F}_{\vdash}(\mathcal{S})\right)$ there is an $a \in \mathcal{A}$ with $\operatorname{Conc}(a)=\phi$.

Where the setting $\mathcal{A} \mathcal{F}_{\vdash}$ is clear from the context we will simply write $\sim_{\text {Sem }}$ to avoid clutter.

Remark 2. For reasons of space we restrict our focus in this paper on skeptical consequence as defined in Definition 6 . Note that $\sim_{\mathrm{Cmp}}^{\mathcal{A F} \mathcal{F}_{\vdash}}$ coincides with $\sim_{\mathrm{Grd}}^{\mathcal{A \mathcal { F }} \vdash}$.

\section{The Relevance Properties}

\subsection{Syntactic Relevance}

A syntactical relevance property that has been proposed in the context of structured argumentation is noninterference [Caminada et al., 2011]. Let us call two sets of formulas syntactically disjoint if no atom that occurs in a formula in $\mathcal{S}_{1}$ also occurs in a formula in $\mathcal{S}_{2}$ and vice versa: so $\operatorname{Atoms}\left(\mathcal{S}_{1}\right) \cap \operatorname{Atoms}\left(\mathcal{S}_{2}\right)=\emptyset$ where $\operatorname{Atoms}(\mathcal{S})$ is the set of atoms occurring in formulas in $\mathcal{S}$. In such cases we write: $\mathcal{S}_{1} \mid \mathcal{S}_{2}$.

Definition 7 (Non-Interference, [Caminada et al., 2011]). $\sim \subseteq \wp(\mathcal{L}) \times \mathcal{L}$ satisfies Non-Interference iff for all $S_{1} \cup$ $\{\phi\} \cup \mathcal{S}_{2} \subseteq \mathcal{L}$ for which $\left(\mathcal{S}_{1} \cup\{\phi\}\right) \mid \mathcal{S}_{2}$ we have: ${ }^{1}$ $\mathcal{S}_{1} \sim \phi$ iff $\mathcal{S}_{1} \cup \mathcal{S}_{2} \sim \phi$.

Definition 8 (Contamination, [Caminada et al., 2011]). Let $\sim \subseteq \wp(\mathcal{L}) \times \mathcal{L}$ be a consequence relation. A set $\mathcal{S} \subseteq \mathcal{L}$, such that $\operatorname{Atoms}(\mathcal{S}) \subset \operatorname{Atoms}(\mathcal{L})$, is called contaminating (with respect to $(\mathcal{)})$, if for any set of formulas $\mathcal{S}^{\prime} \subseteq \mathcal{L}$ such that $\mathcal{S} \mid \mathcal{S}^{\prime}$ and for every $\phi \in \mathcal{L}$, it holds that $\mathcal{S} \sim \phi$ if and only if $\mathcal{S} \cup \mathcal{S}^{\prime} \sim \phi$.

Consequence relations that are non-trivial and satisfy NonInterference also satisfy Crash-Resistance: ${ }^{2}$

Definition 9 (Crash-Resistance, [Caminada et al., 2011]). A consequence relation $\sim \subseteq \wp(\mathcal{L}) \times \mathcal{L}$ satisfies CrashResistance iff there is no set $\mathcal{S} \subseteq \mathcal{L}$ that is contaminating with respect to $\sim$.

Given a setting $\mathcal{A F}_{\vdash}$, a natural question is whether NonInterference is a property that gets inherited on the level of non-monotonic inference $\sim_{\text {sem }}$ from $\vdash$ : we will show below that in case $\vdash$ satisfies Non-Interference so does $\sim_{\text {Sem }}$. In fact, the following less requiring criterion is sufficient:

Definition 10 (Pre-Relevance). $\vdash \subseteq \wp(\mathcal{L}) \times \mathcal{L}$ satisfies PreRelevance iff for all $\mathcal{S}_{1} \cup\{\phi\} \cup \mathcal{S}_{2} \subseteq \mathcal{L}$ for which $\mathcal{S}_{1} \cup\{\phi\}$ $\mathcal{S}_{2}$ : if $\mathcal{S}_{1} \cup \mathcal{S}_{2} \vdash \phi$ then there is a $\mathcal{S}_{1}^{\prime} \subseteq \mathcal{S}_{1}$ such that $\mathcal{S}_{1}^{\prime} \vdash \phi$.

When considering attacks we need to extend the notion of Pre-Relevance by taking into account $\widehat{\cdot}$ and $\tau$. We first define:

Definition 11 (Prime settings). A setting $(\vdash,-, \cdot)$ is prime iff for all sets of atoms $\mathcal{A}_{1}$ and $\mathcal{A}_{2}$ in $\mathcal{L}$ for which $\mathcal{A}_{1} \mid \mathcal{A}_{2}$, for all $\mathcal{S}_{1}, \mathcal{T}_{1}, \mathcal{S}_{2}, \mathcal{T}_{2} \in \wp_{\text {fin }}(\mathcal{L})$ for which $\operatorname{Atoms}\left(\mathcal{S}_{1}\right), \operatorname{Atoms}\left(\mathcal{T}_{1}\right) \subseteq$ $\mathcal{A}_{1}$ and $\operatorname{Atoms}\left(\mathcal{S}_{2}\right)$, Atoms $\left(\mathcal{T}_{2}\right) \subseteq \mathcal{A}_{2}$, and for all $\phi$ and $\psi$ for which $\psi \in \bar{\phi}$ and $\phi \in \widehat{\mathcal{T}_{1} \cup \mathcal{T}_{2}}$, we have:

if $\mathcal{S}_{1} \cup \mathcal{S}_{2} \vdash \psi$ then there are $i \in\{1,2\}, \mathcal{S}_{i}^{\prime} \subseteq \mathcal{S}_{i}, \phi_{i} \in \widehat{\mathcal{T}}_{i}$ and $\psi_{i} \in \overline{\phi_{i}}$ for which $\mathcal{S}_{i}^{\prime} \vdash \psi_{i}$.

Definition 12 (Pre-Relevant Settings). A setting $\mathcal{A F}=$ $\left(\vdash, \overline{,}, \hat{\ominus}^{\circ}\right)$ is Pre-Relevant iff (i) $\vdash$ is Pre-Relevant, (ii) $\mathcal{A F}$ is prime, and (iii) $\widehat{\cdot}$ is $\subseteq$-monotonic (i.e., for all $\Delta, \Delta^{\prime} \in$ $\left.\wp_{\text {fin }}(\mathcal{L}), \widehat{\Delta} \subseteq \widehat{\widehat{U \cup \Delta^{\prime}}}\right)$.

Example 3. Note that $\widehat{\cdot}: \Delta \mapsto \Delta$ (see Ex. 1) and $\widehat{\imath}: \Delta \mapsto$ $\left\{\wedge \Delta^{\prime} \mid \emptyset \subset \Delta^{\prime} \subseteq \Delta\right\}$ (see Ex. 2) are both $\subseteq$-monotonic.

Fact 1. Where $\widehat{\cdot}=\mathrm{id}(\cdot)($ see Ex. 1) and $\bar{\gamma}=\{\neg \gamma\}$, the PreRelevance of $(\vdash,-, \cdot)$ follows from the Pre-Relevance of $\vdash$.

Proof. Items (i) and (iii) are trivial. For Item (ii) suppose that $\mathcal{S}_{1} \cup \mathcal{S}_{2} \vdash \psi$, where $\psi=\neg \phi$ and $\phi \in \widehat{\mathcal{T}_{1} \cup \mathcal{T}_{2}}=\mathcal{T}_{1} \cup \mathcal{T}_{2}$ and where $\mathcal{S}_{1}, \mathcal{S}_{2}, \mathcal{T}_{1}, \mathcal{T}_{2}$ are as in Def. 11. Thus, there is an $i \in\{1,2\}$ s.t. $\phi \in \mathcal{T}_{i}$. Thus, Atoms $(\psi) \subseteq \mathcal{A}_{i}$. By the PreRelevance of $\vdash$, there is an $\mathcal{S}_{i}^{\prime} \subseteq \mathcal{S}_{i}$ for which $\mathcal{S}_{i}^{\prime} \vdash \psi$.

${ }^{1}$ A similar property is Basic Relevance [Avron, 2014, Definition 3.1].

${ }^{2} \sim$ is non-trivial if there are always two sets of formulas with the same atoms but different conclusions (see [Caminada et al., 2011]). 
Fact 2. Where $\widehat{\Delta}=\left\{\bigwedge \Delta^{\prime} \mid \emptyset \subset \Delta^{\prime} \subseteq \Delta\right\}$ (see Ex. 2), $\bar{\gamma}=$ $\{\neg \gamma\}$ and $\vdash$ is contrapositable (i.e., $\mathcal{S} \vdash \neg \Lambda\left(\Delta \cup \Delta^{\prime}\right)$ implies $\left.\mathcal{S} \cup \Delta^{\prime} \vdash \neg \Lambda \Delta\right)$, the Pre-Relevance of $(\vdash,-, \cdot)$ follows from the Pre-Relevance of $\vdash$.

In Section 5.1 we will show that:

Theorem 1. If $\mathcal{A} \mathcal{F}_{\vdash}$ satisfies Pre-Relevance then $\sim_{\text {Sem }}^{\mathcal{A} \mathcal{F}_{\vdash}}$ satisfies Non-Interference for each Sem $\in\{\mathrm{Grd}$, Prf $\}$.

Example 4. We take the setting $\mathcal{A F}_{\vdash_{\mathrm{RM}}}=\left(\vdash_{\mathrm{RM}}, \overline{,}\right.$, id $)$, where $\vdash_{\mathrm{RM}}$ is the consequence relation of the semi-relevance logic $\mathrm{RM}$ and $:: \phi \mapsto\{\neg \phi\}$. $\vdash_{\mathrm{RM}}$ satisfies Pre-Relevance (see [Avron, 2016, Prop. 6.5]) and thus $\mathcal{A F}_{\vdash_{\mathrm{RM}}}$ satisfies NonInference and Crash-Resistance. Similar for other relevance logics.

Example 5. Although $\vdash_{\mathrm{CL}}$ does not satisfy Pre-Relevance, $\vdash_{\mathrm{CL}}^{\top}$ does, where $\vdash_{\mathrm{CL}}^{\top}$ is the restriction of $\vdash_{\mathrm{CL}}$ to pairs $(\Gamma, \gamma)$ for which $\nvdash_{\mathrm{CL}} \neg \bigwedge \Gamma$. Hence, $\mathcal{A} \mathcal{F}_{\vdash_{\mathrm{CL}}^{\top}}=\left(\vdash_{\mathrm{CL}}^{\top},{ }^{\circ}\right.$, id $)$ where : : $\phi \mapsto\{\neg \phi\}$ satisfies Non-Interference. In [Wu and Podlaszewski, 2014] such a restriction is applied in the context of ASPIC.

Example 6. Recently paraconsistent logics based on maximal consistent subsets [Grooters and Prakken, 2016] have been used in the context of structured argumentation. Let $\Gamma \vdash_{\text {mcs }}^{n} \phi$ $\left[\Gamma \vdash\right.$ mcs $\phi$ ] iff for all [some] maximal consistent subsets $\Gamma^{\prime}$ of $\Gamma, \Gamma^{\prime} \vdash_{\mathrm{CL}} \phi$. ( $\Gamma^{\prime} \subseteq \Gamma$ is a maximal consistent subset of $\Gamma$ if it is consistent and there are no consistent $\Gamma^{\prime \prime} \subseteq \Gamma$ such that $\Gamma^{\prime} \subset \Gamma^{\prime \prime}$.) Such consequence relations satisfy Pre-Relevance and thus, argumentative settings based on them satisfy NonInterference.

A refinement of Theorem 1 is given in Corollary 1 below.

Definition 13. Given a setting $(\vdash,,, \uparrow)$ let $\vdash^{\emptyset}$ be the restriction of $\vdash$ to pairs $(\Gamma, \gamma)$ for which there is no $(\emptyset, \delta) \in \vdash$ such that $\delta \in \bar{\psi}$ for some $\psi \in \widehat{\Gamma}$. have:

Since arguments with empty supports have no attackers we

Lemma 1. Where Sem $\in\{$ Grd, $\operatorname{Prf}\}$ and $\mathcal{S} \subseteq \mathcal{L}$,

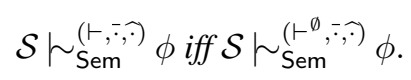

Corollary 1. If $\mathcal{A F}_{\vdash \emptyset}$ satisfies Pre-Relevance then $\sim_{\mathrm{Sem}}^{\mathcal{A} \mathcal{F}_{\vdash}}$ satisfies Non-Interference for each Sem $\in\{$ Grd, Prf $\}$.

We illustrate the latter point with an example.

Example 7. Also the setting $\mathcal{A F}_{F_{\mathrm{CL}}}^{\text {def }}$ in Ex. 2 satisfies NonInterference. Note for this that $\vdash_{\mathrm{CL}}^{\emptyset}=\vdash_{\mathrm{CL}}^{\top}$ (where the latter is defined as in Ex. 5) in the context of $\mathcal{A F}_{\vdash \mathrm{CL}}^{\text {def }}$.

In the following sections we will relate these results to systems of structured argumentation from the literature.

\subsection{Semantic Relevance}

As for semantic relevance we study in this paper a criterion known from non-monotonic logic, namely Cumulativity.

Definition 14. Given $\vdash \subseteq \wp(\mathcal{L}) \times \mathcal{L}$ and $\phi \in \mathcal{L}$, let $\vdash^{+\phi}$ be the transitive closure of $\vdash \cup\{(\emptyset, \phi)\}$. Given a setting $\mathcal{A} \mathcal{F}_{\vdash}$ and a semantics Sem $\in\{$ Grd, Prf $\}$ let $\sim_{\text {Sem }}^{+\phi}$ be an abbreviation of $\sim_{\text {Sem }}^{\mathcal{A F}_{++\phi}}$ and $\mathcal{A} \mathcal{F}_{\vdash}^{+\phi}$ for $\mathcal{A F}_{\vdash+\phi}$.
On the level of consequence relations Cumulativity is the following property, intuitively expressing that the consequence set is invariant under the addition of derivable formulas to the premises:

Definition 15 (Cumulativity). A setting $\mathcal{A F}_{\vdash}$ satisfies $\mathrm{Cu}$ mulativity for Sem $\in\{$ Grd, Prf $\}$, iff, for all $\mathcal{S} \cup\{\phi, \psi\} \subseteq \mathcal{L}$ such that $\mathcal{S} \sim_{\text {Sem }} \phi$ we have: $\mathcal{S} \sim_{\text {Sem }}^{+\phi} \psi$ iff $\mathcal{S} \sim_{\text {Sem }} \psi$.

On the level of Dung-extensions, Cumulativity is:

Definition 16 (Extensional Cumulativity). A setting $\mathcal{A F}_{\vdash}$ satisfies Extensional Cumulativity for Sem $\in\{$ Grd,Prf $\}$ iff for all $\mathcal{S} \cup\{\phi\} \subseteq \mathcal{L}$ such that $\mathcal{S} \sim_{\text {sem }} \phi$ we have: $\operatorname{Sem}\left(\mathcal{A F}_{\vdash}(\mathcal{S})\right)=\left\{\mathcal{E} \cap \operatorname{Arg}_{\vdash}(\mathcal{S}) \mid \mathcal{E} \in \operatorname{Sem}\left(\mathcal{A F}_{\vdash+\phi}(S)\right)\right\}$.

We will show, in Section 5.2, that a setting $\mathcal{A} \mathcal{F}_{\vdash}$ satisfies Cumulativity for grounded semantics if $\mathcal{A F}_{\vdash}$ is pointed:

Definition 17 (Pointed Settings). $(\vdash, \cdot, \cdot)$ is pointed iff

1. for all $\Gamma, \Delta \in \wp_{\text {fin }}(\mathcal{L}), \widehat{\Gamma \cup \Delta}=\widehat{\Gamma} \cup \widehat{\Delta}$ (in this case we say that $\hat{r}$ is pointed), and

2. $\vdash$ satisfies Cut w.r.t. $\vdash^{+\phi}$ for any $\phi \in \mathcal{L}$, i.e., for every $\Gamma \cup\{\gamma\} \subseteq \mathcal{L}, \Gamma \cup \Delta \vdash \gamma$ if $\Gamma \vdash \phi$ and $\Delta \vdash^{+\phi} \gamma$.

Theorem 2. Where $\mathcal{A F}_{\vdash}$ is pointed, $\mathcal{A F}_{\vdash}$ satisfies Cumulativity and Extensional Cumulativity for grounded semantics.

Example 8. Any setting $(\vdash, \overline{,}$, id) is pointed iff $\vdash$ satisfies Cut. For instance, each of the consequence relations in Examples 1 and 4 satisfies Cut and thus the corresponding settings are pointed and therefore satisfy Cumulativity.

If we restrict $\vdash$ to consistent sets on the left side, denoted by $\vdash_{\text {con }}$ (see Def. 19 below) and if $\vdash$ satisfies Cut and Contraposition (see Def. 18 below), then the setting $\mathcal{A} \mathcal{F}_{\text {con }}=\left(\vdash_{\text {con }}\right.$, $\bar{r}$, id) is cumulative. In more detail:

Definition 18. $(\vdash,-)$ is contrapositable iff for all $\Theta \in$ $\wp_{\text {fin }}(\mathcal{L})$, if $\Theta \vdash \gamma^{\prime}$ where $\gamma^{\prime} \in \bar{\gamma}$ then for all $\sigma \in \Theta$, $(\Theta \cup\{\gamma\}) \backslash\{\sigma\} \vdash \sigma^{\prime}$ for some $\sigma^{\prime} \in \bar{\sigma}$. By extension we call $\mathcal{A F}_{\vdash}=\langle\vdash,-, \cdot\rangle$ contrapositable if $(\vdash,-)$ is contrapositable.

Definition 19. Where $\mathcal{A} \mathcal{F}_{\vdash}=\langle\vdash, \overline{,}$, id $\rangle$, a set $\Theta \subseteq \mathcal{S}$ is $\mathcal{A} \mathcal{F}_{\vdash}(\mathcal{S})$-inconsistent iff there is a $\Theta^{\prime} \subseteq \Theta$ and a $\gamma \in \Theta^{\prime}$ for which $\Theta \backslash\{\gamma\} \vdash \gamma^{\prime}$ where $\gamma^{\prime} \in \bar{\gamma}$. $\Theta$ is $\mathcal{A} \mathcal{F}_{\vdash}(\mathcal{S})$-consistent iff it is not $\mathcal{A} \mathcal{F}_{\vdash}(\mathcal{S})$-inconsistent.

Given $\vdash$, let $\vdash_{\text {con }}=\left\{(\Gamma, \gamma) \mid \Gamma \vdash \gamma\right.$ and $\Gamma$ is $\mathcal{A F}_{\vdash}$-consistent $\}$.

Theorem 3. Where $\mathcal{A} \mathcal{F}_{\vdash}=(\vdash, \overline{,}$, id $)$ is contrapositable and $\vdash$ satisfies Cut, $\mathcal{A F}_{\text {con }}=\left(\vdash_{\mathrm{con}},{ }^{-}\right.$, id $)$is cumulative and extensionally cumulative for $\mathrm{Sem} \in\{\mathrm{Grd}, \mathrm{Prf}, \mathrm{Stb}\}$.

Example 9. In view of Theorem 3, $\mathcal{A F}_{\vdash_{\mathrm{CL}}^{\top}}$ from Ex. 5 is cumulative for Sem $\in\{$ Grd, Stb, Prf $\}$.

\section{Systems of Structured Argumentation}

In this section we take a look at several of the structured argumentation frameworks from the literature and show how they can be represented in our setting.

Example 10 (Logic-Based Argumentation). Logic-based argumentation is closest to our setting from Section 2. Systems can be found in, for instance, [Arieli and Straßer, 2015; 
Besnard and Hunter, 2014]. ${ }^{3}$ The core logic L is a finitary Tarskian logic with an adequate consequence relation $\vdash \subseteq \wp_{\text {fin }}(\mathcal{L}) \times \mathcal{L}$. Given a set $\mathcal{S} \subseteq \mathcal{L}$, the set of arguments defined by $\operatorname{Arg}_{\vdash}(\mathcal{S})$ consists of all $(\Gamma, \gamma)$ where $\Gamma \vdash \gamma$ and $\Gamma \subseteq \mathcal{S}$ just like in Def. 1. Different attack rules have been proposed, such as: $(\Gamma, \gamma)$ attacks $(\Delta, \psi)$ iff ...

Defeat (Def): $\quad \gamma \vdash \neg \bigwedge \Delta^{\prime}$ for some $\emptyset \neq \Delta^{\prime} \subseteq \Delta$.

Undercut (Ucut): $\vdash \gamma \equiv \neg \bigwedge \Delta^{\prime}$ for some $\emptyset \neq \Delta^{\prime} \subseteq \Delta$.

Direct Compact Defeat (DiCoDef): $\gamma=\neg \delta^{\prime}$ for some $\delta^{\prime} \in \Delta$.

Direct Undercut (DiUcut): $\quad$ there is a $\delta \in \Delta$ s.t. $\vdash \gamma \equiv \neg \delta$.

Direct Defeat (DiDef): $\quad$ there is a $\delta \in \Delta$ s.t. $\gamma \vdash \neg \delta$.

Dung semantics are defined as usual on top of an attack diagram analogous to Definitions 4 and 5. Consequence relations are defined analogous to Definition $6 \mathcal{S} \| \sim_{\text {Sem }} \phi$ iff in all Sem-extensions there is an argument $(\Gamma, \phi)$.

Systems of logic-based argumentation translate rather directly to our setting. We only need to adjust the definitions of $\cdot$ and $\cdot$ so that we can use our attack definition to simulate the attack definitions above. The following table shows how:

\begin{tabular}{lcc} 
& $\bar{\delta}$ & $\widehat{\Delta}$ \\
\hline DiCoDef & $\{\neg \delta\}$ & $\Delta$ \\
Def & $\{\neg \delta\}$ & $\left\{\bigwedge \Delta^{\prime} \mid \emptyset \subset \Delta^{\prime} \subseteq \Delta\right\}$ \\
DiDef & $\{\gamma \mid \gamma \vdash \neg \delta\}$ & $\Delta$ \\
DiUcut & $\{\gamma \mid \gamma \vdash \neg \delta, \neg \delta \vdash \gamma\}$ & $\Delta$ \\
Ucut & $\{\gamma \mid \gamma \vdash \neg \delta, \neg \delta \vdash \gamma\}$ & $\left\{\bigwedge \Delta^{\prime} \mid \emptyset \subset \Delta^{\prime} \subseteq \Delta\right\}$
\end{tabular}

The easy proof concerning the adequacy of our representations is omitted for reasons of space.

Remark 3. The definitions for direct attack forms (DiDef, DiUcut, DiCoDef) all give rise to a pointed $\cdot$ (namely id) in our representation. Thus, combining these attack forms with core logics $L$ for which $\vdash_{\mathrm{L}}$ satisfies Cut, we obtain Cumulativity.

Remark 4. Instantiating logic-based argumentation with a core logic that satisfies Pre-Relevance (such as the ones in Examples 4, 5, 6) we obtain Non-Interference.

Example 11 (Assumption-Based Argumentation (ABA), [Bondarenko et al., 1997]). Let $\mathcal{L}$ be a formal language, : : $\mathcal{L} \rightarrow \wp(\mathcal{L})$ a contrariness function, $A b \subseteq \mathcal{L}$ a subset of so-called assumptions, and $\mathcal{R}$ be a set of rules of the form $\phi_{1}, \ldots, \phi_{n} \rightarrow \phi$ where $\phi_{1}, \ldots, \phi_{n}, \phi \in \mathcal{L}$ and $\phi \notin A b .{ }^{4}$ There is an $\mathcal{R}$-deduction from some $\Delta \subseteq A b$ to $\phi$ iff there is a sequence $\phi_{1}, \ldots, \phi_{n}$ for which $\Delta=\left\{\phi_{1}, \ldots, \phi_{n}\right\} \cap A b$, $\phi_{n}=\phi$ and for each $1 \leq i \leq n, \phi_{i}$ is either in $\Delta$ or there is a rule $\phi_{i_{1}}, \ldots, \phi_{i_{m}} \rightarrow \bar{\phi}_{i}$ where $i_{1}, \ldots, i_{m}<i$. Given two sets of assumptions $\Delta, \Delta^{\prime} \subseteq A b, \Delta$ attacks $\Delta^{\prime}$ iff there is a $\delta \in \Delta^{\prime}$ for which there is an $\mathcal{R}$-deduction of some $\psi \in \bar{\delta}$ from

\footnotetext{
${ }^{3}$ There are differences between these presentations: while [Besnard and Hunter, 2014] uses classical logic as a core logic, [Arieli and Straßer, 2015] allows for any Tarskian logic with an adequate sequent calculus to serve as core logic. [Besnard and Hunter, 2014] requires the support sets of arguments to be consistent and minimal while [Arieli and Straßer, 2015] omit this requirement. In what follows we follow the generalized setting of [Arieli and Straßer, 2015]. Consistency and minimality can easily be captured by changing the underlying relation $\vdash$ (see e.g., Ex. 5).

${ }^{4}$ In this paper we restrict ourselves to so-called flat frameworks that satisfy the latter requirement.
}

some $\Delta^{\prime \prime} \subseteq \Delta$. Subsets of assumptions in $A b$ and attacks between them give rise to an attack diagram where nodes are sets of assumptions and arcs are attacks. Dung-style semantics are applied to these graphs: $\Delta$ is conflict-free if it does not attack itself, $\Delta$ is admissible if it defends itself, it is complete if it contains all assumptions it defends, it is preferred if it is maximally admissible and stable if it is admissible and attacks every assumption it does not contain. Given a semantics Sem, a consequence relation is given by $(A b, \mathcal{R}) \| \sim \sim_{\mathrm{Sem}}^{\text {aba }} \phi$ iff $\phi$ is $\mathcal{R}$-derivable from all sets of assumptions $\Delta \subseteq A b$ that satisfy the requirements of Sem.

In most presentations of ABA, the rules $\mathcal{R}$ are considered domain-specific strict inference rules that are part of a given knowledge base. They may also be obtained from an underlying core logic $\mathrm{L}$ with consequence relation $\vdash_{\mathrm{L}}$ by setting $\phi_{1}, \ldots, \phi_{n} \rightarrow \phi$ iff $\left\{\phi_{1}, \ldots, \phi_{n}\right\} \vdash_{\mathrm{L}} \phi$.

We can translate ABA into our setting as follows. Where $\mathcal{R}$ represents domain-specific rules that are part of the knowledge base, we define for $\Delta \subseteq A b$ and $\mathcal{R}^{\prime} \subseteq \mathcal{R}$ :

(†aba) $\Delta \cup \mathcal{R}^{\prime} \vdash \phi$, iff, there is an $\mathcal{R}$-deduction of $\phi$ from $\Delta$ making use of the rules in $\mathcal{R}^{\prime}$ (and only of these). ${ }^{5}$

Where $\mathcal{R}$ is generated from a given core logic $\mathrm{L}$, we define for $\Delta \subseteq A b$ :

(†aba) $\Delta \vdash \phi$, iff, $\Delta \vdash_{\mathrm{L}} \phi$.

In both cases, we use the definition of $:$ from $\mathrm{ABA}$, let $\widehat{\cdot}=\mathrm{id}(\cdot)$. Clearly, in our setting $(\Delta, \delta)$ attacks $(\Gamma, \gamma)$ iff $\delta \in \bar{\phi}$ for some $\phi \in \Gamma$. For reasons of space we omit the proof that the setting $\mathcal{A F}_{\vdash}(A b \cup \mathcal{R})$ [resp. $\left.\mathcal{A F}_{\vdash}(A b)\right]$ adequately represents the $\mathrm{ABA}$ framework based on $A b$ and $\mathcal{R}$ for $\vdash$ in $\left(\dagger_{\text {aba }}\right)$ [resp. (†aba)] so that $(A b, \mathcal{R}) \| \sim_{\text {Sem }}^{\text {aba }} \phi$ iff $A b \cup \mathcal{R} \sim_{\text {Sem }}^{\mathcal{A} \mathcal{F}_{\vdash}} \phi\left[\right.$ resp. $\left.A b \sim_{\text {Sem }}^{\mathcal{A} \mathcal{F}_{\vdash}} \phi\right]$.

Remark 5. It is easy to see that for representation ( $\left.\dagger_{a b a}\right)$ the underlying consequence relation $\vdash$ satisfies Pre-Relevance and if $(\dagger) \operatorname{Atoms}(\bar{\phi}) \subseteq \operatorname{Atoms}(\phi)$ for all $\phi \in \mathcal{L}$, we obtain Non-Interference. For the representation ( $\left.\ddagger_{a b a}\right)$ it depends on the logic $L$. In case $\vdash_{L}$ satisfies Pre-Relevance and if $(\dagger)$ we obtain Non-Interference.

Remark 6. Our representation of ABA makes use of the pointed $\widehat{\cdot}$ (namely id) and $\mathcal{R}$-derivability satisfies Cut. Note that $\mathcal{A F}_{\vdash+\phi}(A b \cup \mathcal{R})$ [resp. $\left.\mathcal{A F}_{\vdash+\phi}(A b)\right]$ adequately represents the ABA framework based on $(A b, \mathcal{R} \cup\{\rightarrow \phi\})$ for $\vdash$ in $\left(\dagger_{a b a}\right)$ [resp. for $\vdash$ in ( $\left.\left.\ddagger_{a b a}\right)\right]$. Thus we obtain Cumulativity. Example 12 (ASPIC, [Prakken, 2010]). In ASPIC we work with a formal language $\mathcal{L}$, a contrariness function ${ }^{-}: \mathcal{L} \rightarrow$ $\wp(\mathcal{L})$, a set of defeasible rules $\mathcal{D}$ and a set of strict rules $\mathcal{R}$ of the form $A_{1}, \ldots, A_{n} \Rightarrow A$ resp. $A_{1}, \ldots, A_{n} \rightarrow A$. Similarly as was the case for ABA, the strict rules may reflect domainspecific knowledge or be generated in view of an underlying core logic $\mathrm{L}$. We assume that $\mathcal{L}$ contains for each defeasible rule $R \in \mathcal{D}$ a logical atom $n(R)$ that serves as name of $R$. An $(\mathcal{D}, \mathcal{R})$-deduction of $\phi \in \mathcal{L}$ from $\Delta \subseteq \mathcal{L}$ is given by a tree

- whose leaves are labeled by elements in $\Delta$ (so that each $\delta \in \Delta$ occurs as label of a leaf),

\footnotetext{
${ }^{5}$ For this the language $\mathcal{L}$ underlying the original $\mathrm{ABA}$ framework is enriched by $\mathcal{R}$ so that $\vdash \subseteq \wp_{\text {fin }}(\mathcal{L} \cup \mathcal{R}) \times \mathcal{L}$. This is important to track syntactic relevance.
} 
- for every non-root node labeled by $\psi$ there is a rule $R=$ $\phi_{1}, \ldots, \phi_{n} \rightarrow \psi \in \mathcal{R}$ or $R=\phi_{1}, \ldots, \phi_{n} \Rightarrow \psi \in$ $\mathcal{D}$ and its child-nodes are labeled by $\phi_{1}, \ldots, \phi_{n}$ (if $R$ has an empty body, the single child-node is unlabeled). The edges connecting the child-nodes with the parent are labeled $R .^{6}$

- the root of the tree is labeled by $\phi$.

Given a $(\mathcal{D}, \mathcal{R})$-derivation $a, \operatorname{DefC}(a)[\operatorname{StrC}(a)]$ is the set of all node labels to which an edge labeled with a defeasible [strict] rule leads and $\operatorname{DefR}(a)[\operatorname{StrR}(a)]$ is the set of all edge labels that are defeasible [strict] rules.

An argumentation theory is a triple $(\mathcal{P}, \mathcal{R}, \mathcal{D})$ where $\mathcal{P} \subseteq$ $\mathcal{L}$ is a set of premises, $\mathcal{R}$ is a set of strict rules and $\mathcal{D}$ is a set of defeasible rules. The set $\operatorname{Arg}_{\text {aspic }}(\mathcal{P}, \mathcal{R}, \mathcal{D})$ is the set of all $(\mathcal{D}, \mathcal{R})$-derivations of some $\phi \in \mathcal{L}$ from some finite $\Delta \subseteq \mathcal{P}$. Given two arguments $a, b \in \operatorname{Arg}_{\text {aspic }}(\mathcal{P}, \mathcal{R}, \mathcal{D})$, a rebuts $b$ iff there is a $\phi \in \operatorname{DefC}(b)$ such that $\operatorname{Conc}(a) \in \bar{\phi}$; a undercuts $b$ iff $a \in \overline{n(R)}$ for some $R \in \operatorname{DefR}(b)$. Attack diagrams, underlying Dung-semantics and consequence relations $\| \sim_{\text {Sem }}^{\text {aspic }}$ are then defined in the usual way.

To represent ASPIC in our setting we first need to define our derivability relation and then translate the ASPIC attacks. In case the set of strict rules $\mathcal{R}$ presents domainspecific knowledge we define:

(†aspic $) \Gamma \vdash \phi$ iff there is a $(\mathcal{D}, \mathcal{R})$-derivation $a$ of $\phi$ from $\mathcal{P}$ where $\Gamma=\{R, n(R) \mid R \in \operatorname{DefR}(a)\} \cup \operatorname{DefC}(a) \cup$ $\operatorname{StrR}(a) \cup\{\rightarrow \psi \mid \psi \in \mathcal{P}\}{ }^{7}$

If $\mathcal{R}$ is generated via an underlying core logic we define:

(łaspic) $\Gamma \vdash \phi$ iff there is a $(\mathcal{D}, \mathcal{R})$-derivation $a$ of $\phi$ from $\mathcal{P}$ where $\Gamma=\{R, n(R) \mid R \in \operatorname{DefR}(a)\} \cup \operatorname{DefC}(a) \cup\{\rightarrow$ $\psi \mid \psi \in \mathcal{P}\}$.

For reasons of space we omit the proof that, where $\mathcal{S}=$ $\{R, n(R), \operatorname{Conc}(R) \mid R \in \mathcal{D}\} \cup\{\rightarrow \psi \mid \psi \in \mathcal{P}\}$ and $\widehat{\cdot}=$ id $(\cdot),{ }^{8}$ the setting $\mathcal{A} \mathcal{F}_{\vdash}(\mathcal{S} \cup \mathcal{R})\left[\operatorname{resp} . \mathcal{A} \mathcal{F}_{\vdash}(\mathcal{S})\right]$ represents the ASPIC theory $(\mathcal{P}, \mathcal{R}, \mathcal{D})$ for $\vdash$ in (†aspic $)$ [resp. in ( $\left.\left.\ddagger_{\text {aspic }}\right)\right]$ so that $(\mathcal{P}, \mathcal{R}, \mathcal{D}) \| \sim_{\text {Sem }}^{\text {aspic }} \phi$ iff $\mathcal{S} \cup \mathcal{R} \sim_{\text {Sem }}^{\mathcal{A} \mathcal{F}_{\vdash}} \phi\left[\right.$ resp. $\mathcal{S} \sim_{\text {Sem }_{\vdash}}^{\mathcal{A} \mathcal{F}_{\vdash}} \phi$. Remark 7. Analogous to Remark 5, if ( $\dagger$ ) holds, we obtain Non-Interference for the presentation ( $\left.\dagger_{\text {aspic }}\right)$ and for ( $\left.\ddagger_{\text {aspic }}\right)$ if additionally the underlying logic $L$ satisfies Pre-Relevance. Remark 8. Our representation of ASPIC makes use of the pointed $\widehat{\cdot}$ (namely id) and $(\mathcal{D}, \mathcal{R})$-derivability satisfies Cut. Note that $\mathcal{A F}_{1_{+\phi}}(\mathcal{S} \cup \mathcal{R})$ [resp. $\left.\mathcal{A} \mathcal{F}_{1_{+\phi}}(\mathcal{S})\right]$ adequately represents the ASPIC argumentation theory $(\mathcal{P} \cup\{\phi\}, \mathcal{R}, \mathcal{D})$ for $\vdash$ in $\left(\dagger_{\text {aspic }}\right)$ [resp. for $\vdash$ in (†aspic $\left.)\right]$ and $\mathcal{S}$ as specified in Example 12. Thus we obtain Cumulativity for grounded semantics.

\footnotetext{
${ }^{6}$ Usually edges are not labeled with rules in ASPIC (and so in cases of rules with empty bodies, there are usually no child-nodes either). We introduce these labels since they enable us to define our representation in a simpler way. We also simplify the presentation in that we do not assume there to be defeasible premises.

${ }^{7}$ Similar as in the case of ABA we enrich the language $\mathcal{L}$ for $\vdash$ to track syntactic relevance. See Fn. 5.

${ }^{8}$ For the variants ASPIC $^{-}$[Caminada et al., 2014] and ASPIC $^{\ominus}$ [Heyninck and Straßer, 2017] where rebut is unrestricted we need to add $\operatorname{StrC}(a)$ to $\Gamma$ in $\left(\dagger_{\text {aspic }}\right)$ and (†aspic $)$. For generalized rebut in $\mathrm{ASPIC}^{\ominus}$ we can proceed analogous to Ex. 2.
}

\section{Meta-Theory}

We will now present the proofs of Theorems 1 and $2 .^{9}$

\subsection{Syntactic Relevance}

In this section we prove Theorem 1 . In the following we suppose that $\mathcal{A F}_{\vdash}$ is a setting that satisfies Pre-Relevance (see Def. 12). We start with some notations:

Definition 20. Where $\mathcal{S} \subseteq \mathcal{L}$ and $a, b \in \operatorname{Arg}_{\vdash}(\mathcal{S})$, we write $a \preceq b$ iff $\widehat{\operatorname{Supp}(a)} \subseteq \widehat{\operatorname{Supp}(b)}$.

Definition 21. Where $\mathcal{S} \subseteq \mathcal{L}$ and $\mathcal{E} \subseteq \operatorname{Arg}_{\vdash}(\mathcal{S})$, let $\operatorname{Defended}\left(\mathcal{E}, \mathcal{A F}_{\vdash}(\mathcal{S})\right)$ be the set of all arguments $a \in$ $\operatorname{Arg}_{\vdash}(\mathcal{S})$ that are defended by arguments in $\mathcal{E}$.

In view of the monotonicity of $\hat{\imath}$ we have:

Fact 3. Where $b^{\prime} \preceq b$, if a attacks $b^{\prime}$ then a attacks $b$.

Complete extensions are closed under $\preceq$ :

Fact 4. Where $\mathcal{S} \subseteq \mathcal{L}, \mathcal{E} \in \operatorname{Cmp}\left(\mathcal{A} \mathcal{F}_{\vdash}(\mathcal{S})\right)$, $a \in \mathcal{E}$, and $b \in \operatorname{Arg}_{\vdash}(\mathcal{S})$, then $b \in \mathcal{E}$ if $b \preceq a$.

Lemma 2. Where $\mathcal{S} \mid \mathcal{S}^{\prime}$, if $a \in \operatorname{Arg}_{\vdash}\left(\mathcal{S} \cup \mathcal{S}^{\prime}\right)$ attacks $b \in$ $\operatorname{Arg}_{\vdash}(\mathcal{S})$, there is an $a^{\prime} \in \operatorname{Arg}_{\vdash}(\mathcal{S} \cap \operatorname{Supp}(a))$ that attacks $b$.

Proof. Suppose $a=(\Gamma, \psi) \in \operatorname{Arg}_{\vdash}\left(\mathcal{S} \cup \mathcal{S}^{\prime}\right)$ attacks $b=$ $(\Lambda, \sigma) \in A r g_{\vdash}(\mathcal{S})$. Then, $\psi \in \bar{\phi}$ for some $\phi \in \widehat{\Lambda}$. Where $\mathcal{A}_{1}=\operatorname{Atoms}(\mathcal{S}), \mathcal{A}_{2}=\operatorname{Atoms}\left(\mathcal{S}^{\prime}\right), \mathcal{T}_{1}=\Lambda, \mathcal{T}_{2}=\emptyset, \mathcal{S}_{1}=$ $\Gamma \cap \mathcal{S}$ and $\mathcal{S}_{2}=\Gamma \cap \mathcal{S}^{\prime}$, with Def. $11, \mathcal{S}_{1}^{\prime} \vdash \psi^{\prime}$ where $\mathcal{S}_{1}^{\prime} \subseteq \mathcal{S}_{1}$, $\psi^{\prime} \in \overline{\phi^{\prime}}$ and $\phi^{\prime} \in \widehat{\Lambda}$. Thus, $\left(\mathcal{S}_{1}^{\prime}, \psi^{\prime}\right) \preceq a$ attacks $b$.

Lemma 3. Where $\mathcal{S} \mid \mathcal{S}^{\prime}, \mathcal{E} \in \operatorname{Cmp}\left(\mathcal{A} \mathcal{F}_{\vdash}(\mathcal{S})\right), \mathcal{E}^{\prime} \in$ $\operatorname{Cmp}\left(\mathcal{A F}_{\vdash}\left(\mathcal{S}^{\prime}\right)\right), \mathcal{E} \cup \mathcal{E}^{\prime} \in \operatorname{Adm}\left(\mathcal{A F}_{\vdash}\left(\mathcal{S} \cup \mathcal{S}^{\prime}\right)\right)$.

Proof. Suppose $\mathcal{S} \mid \mathcal{S}^{\prime}, \mathcal{E} \in \operatorname{Cmp}\left(\mathcal{A} \mathcal{F}_{\vdash}(\mathcal{S})\right)$ and $\mathcal{E}^{\prime} \in$ $\operatorname{Cmp}\left(\mathcal{A F}_{\vdash}\left(\mathcal{S}^{\prime}\right)\right)$. We now show that $\mathcal{E} \cup \mathcal{E}^{\prime}$ is admissible.

Conflict-free: Assume for a contradiction that there are $a, a^{\prime} \in \mathcal{E} \cup \mathcal{E}^{\prime}$ such that $a$ attacks $a^{\prime}$. By the conflict-freeness of $\mathcal{E}$ and $\mathcal{E}^{\prime}$ it is not the case that $a, a^{\prime} \in \mathcal{E}$ or $a, a^{\prime} \in \mathcal{E}^{\prime}$. Wlog. suppose $a \in \mathcal{E}$ and $a^{\prime} \in \mathcal{E}^{\prime}$. By Lemma 2, there is a $b \in \operatorname{Arg}_{\vdash}\left(\mathcal{S}^{\prime} \cap \operatorname{Supp}(a)\right)=A r g_{\vdash}(\emptyset)$ that attacks $a^{\prime}$. Thus, $b$ is trivially defended by $\mathcal{E}^{\prime}$ and by the completeness of $\mathcal{E}^{\prime}$, $b \in \mathcal{E}^{\prime}$. This is a contradiction to the conflict-freeness of $\mathcal{E}^{\prime}$. Admissibility: Suppose some $b \in \operatorname{Arg}_{\vdash}\left(\mathcal{S} \cup \mathcal{S}^{\prime}\right)$ attacks some $a \in \mathcal{E} \cup \mathcal{E}^{\prime}$. Wlog. assume $a \in \mathcal{E}$. By Lemma 2, there is a $b^{\prime} \in \operatorname{Arg}_{\vdash}(\mathcal{S} \cap \operatorname{Supp}(b))$ that attacks $a$. Thus, there is a $c \in \mathcal{E}$ that attacks $b^{\prime}$. By Fact 3, $c$ attacks $b$.

Lemma 4. Where $\mathcal{S}_{1} \mid \mathcal{S}_{2}, a, b \in \operatorname{Arg}_{\vdash}\left(\mathcal{S}_{1} \cup \mathcal{S}_{2}\right)$, $\operatorname{Supp}(b)=$ $\Theta$ and $b$ attacks $a$,

1. some $b^{\prime} \in \operatorname{Arg}_{\vdash}\left(\mathcal{S}_{1} \cap \Theta\right) \cup \operatorname{Arg}_{\vdash}\left(\mathcal{S}_{2} \cap \Theta\right)$ attacks $a$;

2. if $a \in \operatorname{Arg}_{\vdash}\left(\mathcal{S}_{1}\right)$, some $b^{\prime} \in \operatorname{Arg}_{\vdash}\left(\mathcal{S}_{1} \cap \Theta\right)$ attacks $a$.

Proof. Let $a=(\Gamma, \alpha) \in \operatorname{Arg}_{\vdash}\left(\mathcal{S}_{1} \cup \mathcal{S}_{2}\right)$. Suppose $b=(\Theta, \beta)$ attacks $a$. Thus, there is a $\gamma \in \widehat{\Gamma}$ s.t. $\beta \in \bar{\gamma}$. By Def. 12 (ii), we have $i \in\{1,2\}, \Theta^{\prime} \subseteq \Theta \cap \mathcal{S}_{i}, \phi \in \widehat{\Gamma \cap \mathcal{S}_{i}}$ and $\psi \in \bar{\phi}$ s.t. $b^{\prime}=\left(\Theta^{\prime}, \psi\right) \in \operatorname{Arg}_{\vdash}\left(\mathcal{S}_{i}\right)$. By Def. 12 (iii), $\widehat{\Gamma \cap \mathcal{S}_{i}} \subseteq \widehat{\Gamma}$ and hence $b^{\prime}$ attacks $a$. For Item 2 note that $i=1$ when setting $\mathcal{T}_{1}=\Gamma$ and $\mathcal{T}_{2}=\emptyset$ in Def. 11.

\footnotetext{
${ }^{9}$ The proof of Theorem 3 is omitted for reasons of space.
} 
Lemma 5. Where $\mathcal{S} \mid \mathcal{S}^{\prime}, \mathcal{E} \in \operatorname{Cmp}\left(\mathcal{A F}_{\vdash}\left(\mathcal{S} \cup \mathcal{S}^{\prime}\right)\right)$, $\mathcal{E}_{1}=$ $\mathcal{E} \cap \operatorname{Arg}_{\vdash}(\mathcal{S})$ and $\mathcal{E}_{2}=\mathcal{E} \cap \operatorname{Arg}_{\vdash}\left(\mathcal{S}^{\prime}\right)$,

1. $\mathcal{E}=\operatorname{Defended}\left(\mathcal{E}_{1} \cup \mathcal{E}_{2}, \mathcal{A F}_{\vdash}\left(\mathcal{S} \cup \mathcal{S}^{\prime}\right)\right)$;

2. $\mathcal{E}_{1} \in \mathrm{Cmp}\left(\mathcal{A} \mathcal{F}_{\vdash}(\mathcal{S})\right)$.

Proof. Ad 1. Suppose $\mathcal{E}$ defends some $a \in \operatorname{Arg}_{\vdash}\left(\mathcal{S} \cup \mathcal{S}^{\prime}\right)$. By Lemma 4 and Fact $4, \mathcal{E}_{1} \cup \mathcal{E}_{2}$ defends $a$.

Ad 2. Note that $\mathcal{E}_{1}$ is conflict-free since $\mathcal{E}$ is conflict-free. Suppose $b \in \operatorname{Arg}_{\vdash}(\mathcal{S})$ attacks some $a \in \mathcal{E}_{1}$. Thus, there is a $c \in \mathcal{E}$ that attacks $b$. By Lemma 4 and Fact $4, \mathcal{E}_{1}$ attacks $b$. Thus, $\mathcal{E}_{1}$ is admissible. Suppose $\mathcal{E}_{1}$ defends some $d \in$ $\operatorname{Arg}_{\vdash}(\mathcal{S})$. Then $\mathcal{E}$ defends $d$ and hence $d \in \mathcal{E} \cap \operatorname{Arg}_{\vdash}(\mathcal{S})=$ $\mathcal{E}_{1}$. Hence, $\mathcal{E}_{1}$ is complete.

Lemma 6. Where $\mathcal{S} \mid \mathcal{S}^{\prime}, \mathcal{E}_{1} \in \operatorname{Cmp}\left(\mathcal{A F}_{\vdash}(\mathcal{S})\right)$, $\mathcal{E}_{2} \in$ $\mathrm{Cmp}\left(\mathcal{A F}_{\vdash}\left(\mathcal{S}^{\prime}\right)\right), \mathcal{E}=\operatorname{Defended}\left(\mathcal{E}_{1} \cup \mathcal{E}_{2}, \mathcal{A F}_{\vdash}\left(\mathcal{S} \cup \mathcal{S}^{\prime}\right)\right)$,

1. $\mathcal{E} \cap \operatorname{Arg}_{\vdash}(\mathcal{S})=\mathcal{E}_{1}$ and $\mathcal{E} \cap \operatorname{Arg}_{\vdash}\left(\mathcal{S}^{\prime}\right)=\mathcal{E}_{2}$.

2. $\mathcal{E} \in \mathrm{Cmp}\left(\mathcal{A F}_{\vdash}\left(\mathcal{S} \cup \mathcal{S}^{\prime}\right)\right)$.

Proof. Ad 1. Suppose $a \in \operatorname{Arg}_{\vdash}(\mathcal{S}) \cap \mathcal{E}$. Thus, $a$ it is defended by $\mathcal{E}_{1} \cup \mathcal{E}_{2}$ in $\mathcal{A F}_{\vdash}\left(\mathcal{S} \cup \mathcal{S}^{\prime}\right)$. Suppose some $b \in \operatorname{Arg}_{\vdash}(\mathcal{S})$ attacks $a$. Thus, there is a $c=(\Lambda, \sigma) \in$ $\mathcal{E}_{1} \cup \mathcal{E}_{2}$ that attacks $b$. If $c \in \mathcal{E}_{2}$, by Lemma 2, there is a $c^{\prime} \in \operatorname{Arg}_{\vdash}(\mathcal{S} \cap \Lambda)=\operatorname{Arg}_{\vdash}(\emptyset)$ that attacks $b$. Since $c^{\prime}$ has no attackers, by the completeness of $\mathcal{E}_{1}, c^{\prime} \in \mathcal{E}_{1}$. Altogether this shows that $a \in \operatorname{Defended}\left(\mathcal{E}_{1}, \mathcal{A} \mathcal{F}_{\vdash}\left(\mathcal{S} \cup \mathcal{S}^{\prime}\right)\right)$. Again, by the completeness of $\mathcal{E}_{1}, a \in \mathcal{E}_{1}$. Thus, $\mathcal{E} \cap \operatorname{Arg}_{\vdash}(\mathcal{S})=\mathcal{E}_{1}$. Analogously, $\mathcal{E} \cap \operatorname{Arg}_{\vdash}\left(\mathcal{S}^{\prime}\right)=\mathcal{E}_{2}$. This is Item 1 .

$A d 2$. Suppose there are $a, b \in \mathcal{E}$ such that $a$ attacks $b$. We know that there is a $c \in \mathcal{E}_{1} \cup \mathcal{E}_{2}$ that attacks $a$. Wlog. suppose $c \in \mathcal{E}_{1}$. Thus, there is a $d \in \mathcal{E}_{1} \cup \mathcal{E}_{2}$ that attacks $c$. Since by Lemma $3, \mathcal{E}_{1} \cup \mathcal{E}_{2} \in \operatorname{Adm}\left(\mathcal{A} \mathcal{F}_{\vdash}\left(\mathcal{S} \cup \mathcal{S}^{\prime}\right)\right)$ we have reached a contradiction. Thus, $\mathcal{E}$ is conflict-free.

Suppose now some $a \in \operatorname{Arg}_{\vdash}\left(\mathcal{S} \cup \mathcal{S}^{\prime}\right)$ attacks some $b \in \mathcal{E}$. By the definition of $\mathcal{E}$ there is a $c \in \mathcal{E}_{1} \cup \mathcal{E}_{2}$ that attacks $b$. Thus, $\mathcal{E}$ is admissible.

For completeness assume that $\mathcal{E}$ defends some $a \in$ $\operatorname{Arg}_{\vdash}\left(\mathcal{S} \cup \mathcal{S}^{\prime}\right)$. Suppose $b=(\Lambda, \beta) \in \operatorname{Arg}_{\vdash}\left(\mathcal{S} \cup \mathcal{S}^{\prime}\right)$ attacks $a$. Hence, there is a $c \in \mathcal{E}$ that attacks $b$. In view of Lemma 4 and Fact 3 there is a $c^{\prime} \in\left(\mathcal{E} \cap \operatorname{Arg}_{\vdash}(\mathcal{S})\right) \cup\left(\mathcal{E} \cap \operatorname{Arg}_{\vdash}\left(\mathcal{S}^{\prime}\right)\right)$ that attacks $b$. By Item $1, c^{\prime} \in \mathcal{E}_{1} \cup \mathcal{E}_{2}$ and therefore $a \in \operatorname{Defended}\left(\mathcal{E}_{1} \cup \mathcal{E}_{2}, \mathcal{A F}_{\vdash}\left(\mathcal{S} \cup \mathcal{S}^{\prime}\right)\right)=\mathcal{E}$.

Lemma 7. Where $\mathcal{S} \mid \mathcal{S}^{\prime}, \mathcal{E}_{1} \in \operatorname{Sem}\left(\mathcal{A} \mathcal{F}_{\vdash}(\mathcal{S})\right)$, Sem $\in$ $\{\mathrm{Cmp}, \operatorname{Prf}, \mathrm{Grd}\}$, there is a $\mathcal{E} \in \operatorname{Sem}\left(\mathcal{A F}_{\vdash}\left(\mathcal{S} \cup \mathcal{S}^{\prime}\right)\right)$ for which $\mathcal{E}_{1}=\mathcal{E} \cap \operatorname{Arg}_{\vdash}(\mathcal{S})$.

Proof. (Sem $=\mathrm{Cmp}$ ) Let $\mathcal{E}_{2}$ be arbitrary in $\operatorname{Cmp}\left(\mathcal{A F}_{\vdash}\left(\mathcal{S}^{\prime}\right)\right.$ ). By Lemma $6, \mathcal{E}=\operatorname{Defended}\left(\mathcal{E}_{1} \cup \mathcal{E}_{2}, \mathcal{A F}_{\vdash}\left(\mathcal{S} \cup \mathcal{S}^{\prime}\right)\right) \in$ $\operatorname{Cmp}\left(\mathcal{A F}_{\vdash}\left(\mathcal{S} \cup \mathcal{S}^{\prime}\right)\right)$ and $\mathcal{E}_{1}=\mathcal{E} \cap \operatorname{Arg}_{\vdash}(\mathcal{S})$.

$($ Sem $=$ Grd $)$ Let $\mathcal{E}_{1}=\operatorname{Grd}\left(\mathcal{A} \mathcal{F}_{\vdash}(\mathcal{S})\right), \quad \mathcal{E}_{2}=$ $\operatorname{Grd}\left(\mathcal{A F}_{\vdash}\left(\mathcal{S}^{\prime}\right)\right)$. Again, by Lemma $6, \mathcal{E}=\operatorname{Defended}\left(\mathcal{E}_{1} \cup\right.$ $\left.\mathcal{E}_{2}, \mathcal{A F}_{\vdash}\left(\mathcal{S} \cup \mathcal{S}^{\prime}\right)\right) \in \operatorname{Cmp}\left(\mathcal{A F}_{\vdash}\left(\mathcal{S} \cup \mathcal{S}^{\prime}\right)\right), \mathcal{E}_{1}=\mathcal{E} \cap$ $\operatorname{Arg}_{\vdash}(\mathcal{S})$, and $\mathcal{E}_{2}=\mathcal{E} \cap \operatorname{Arg}_{\vdash}\left(\mathcal{S}^{\prime}\right)$. Suppose there is a $\mathcal{E}^{\star} \subset \mathcal{E}$ such that $\mathcal{E}^{\star} \in \operatorname{Cmp}\left(\mathcal{A} \mathcal{F}_{\vdash}\left(\mathcal{S} \cup \mathcal{S}^{\prime}\right)\right)$. By Lemma $5, \mathcal{E}^{\star} \cap \operatorname{Arg}_{\vdash}(\mathcal{S}) \in \mathrm{Cmp}\left(\mathcal{A F} \mathcal{F}_{\vdash}(\mathcal{S})\right)$ and $\mathcal{E}^{\star} \cap \operatorname{Arg}_{\vdash}\left(\mathcal{S}^{\prime}\right) \in$ $\operatorname{Cmp}\left(\mathcal{A F}_{\vdash}\left(\mathcal{S}^{\prime}\right)\right)$. Thus, $\mathcal{E}^{\star} \cap A \operatorname{Arg}_{\vdash}(\mathcal{S})=\operatorname{Grd}\left(\mathcal{A} \mathcal{F}_{\vdash}(\mathcal{S})\right)$ and $\mathcal{E}^{\star} \cap \operatorname{Arg}_{\vdash}\left(\mathcal{S}^{\prime}\right)=\operatorname{Grd}\left(\mathcal{A F}_{\vdash}\left(\mathcal{S}^{\prime}\right)\right)$. However, by Lemma 5,
$\mathcal{E}^{\star}=\operatorname{Defended}\left(\mathcal{E}_{1} \cup \mathcal{E}_{2}, \mathcal{A} \mathcal{F}_{\vdash}\left(\mathcal{S} \cup \mathcal{S}^{\prime}\right)\right)=\mathcal{E}$, a contradiction with the assumption that $\mathcal{E}^{\star} \subset \mathcal{E}$. The case for preferred extension is similar and left to the reader.

Lemma 8. Where $\mathcal{S} \mid \mathcal{S}^{\prime}, \mathcal{E} \in \operatorname{Prf}\left(\mathcal{A} \mathcal{F}_{\vdash}\left(\mathcal{S} \cup \mathcal{S}^{\prime}\right)\right)$ and $\mathcal{E}_{1}=$ $\mathcal{E} \cap \operatorname{Arg}_{\vdash}(\mathcal{S})$, also $\mathcal{E}_{1} \in \operatorname{Prf}\left(\mathcal{A F}_{\vdash}(\mathcal{S})\right)$.

Proof. Let $\mathcal{S} \mid \mathcal{S}^{\prime}, \mathcal{E} \in \operatorname{Prf}\left(\mathcal{A F} \mathcal{F}_{\vdash}\left(\mathcal{S} \cup \mathcal{S}^{\prime}\right)\right), \mathcal{E}_{1}=\mathcal{E} \cap$ $\operatorname{Arg}_{\vdash}(\mathcal{S})$, and $\mathcal{E}_{2}=\mathcal{E} \cap \operatorname{Arg}_{\vdash}\left(\mathcal{S}^{\prime}\right)$. By Lemma 5, $\mathcal{E}_{1} \in$ $\mathrm{Cmp}\left(\mathcal{A F}_{\vdash}(\mathcal{S})\right)$ and $\mathcal{E}_{2} \in \operatorname{Cmp}\left(\mathcal{A F}_{\vdash}\left(\mathcal{S}^{\prime}\right)\right)$. Let $\mathcal{E}_{1}^{\prime} \in$ $\mathrm{Cmp}\left(\mathcal{A F}_{\vdash}(\mathcal{S})\right)$ for which $\mathcal{E}_{1} \subseteq \mathcal{E}_{1}^{\prime}$. By Lemma 6, where $\mathcal{E}^{\prime}=\operatorname{Defended}\left(\mathcal{E}_{1}^{\prime} \cup \mathcal{E}_{2}, \mathcal{A} \mathcal{F}_{\vdash}\left(\mathcal{S} \cup \mathcal{S}^{\prime}\right)\right), \mathcal{E}^{\prime} \in \operatorname{Cmp}\left(\mathcal{A} \mathcal{F}_{\vdash}(\mathcal{S} \cup\right.$ $\left.\mathcal{S}^{\prime}\right)$ ). By Lemma $5, \mathcal{E}=\operatorname{Defended}\left(\mathcal{E}_{1} \cup \mathcal{E}_{2}, \mathcal{A} \mathcal{F}_{\vdash}\left(\mathcal{S} \cup \mathcal{S}^{\prime}\right)\right)$ and thus $\mathcal{E} \subseteq \mathcal{E}^{\prime}$. Since $\mathcal{E}$ is preferred, $\mathcal{E}=\mathcal{E}^{\prime}$ and hence $\mathcal{E}_{1}=$ $\mathcal{E}_{1}^{\prime}$. Thus, $\mathcal{E}_{1}$ is $\subseteq$-maximal and $\mathcal{E}_{1} \in \operatorname{Prf}\left(\mathcal{A F}_{\vdash}(\mathcal{S})\right)$.

Proof of Theorem 1. Suppose $\mathcal{S} \cup\{\phi\} \mid \mathcal{S}^{\prime}$. We show that $\mathcal{S} \sim_{\text {sem }} \phi$ iff $\mathcal{S} \cup \mathcal{S}^{\prime} \sim_{\text {sem }} \phi . \quad(\Rightarrow)$ Suppose $\mathcal{S} \cup \mathcal{S}^{\prime} \not \chi_{\text {sem }} \phi$. Thus, there is a $\mathcal{E} \in \operatorname{Sem}\left(\mathcal{A} \mathcal{F}_{\vdash}\left(\mathcal{S} \cup \mathcal{S}^{\prime}\right)\right)$ for which there is no $a \in \mathcal{E}$ with conclusion $\phi$. By Lemmas 5 and 8, $\mathcal{E}_{1}=\mathcal{E} \cap \operatorname{Arg}_{\vdash}(\mathcal{S}) \in \operatorname{Sem}\left(\mathcal{A F} \mathcal{F}_{\vdash}(\mathcal{S})\right)$. Since there is no $a \in \mathcal{E}_{1}$ with conclusion $\phi, \mathcal{S} \not \chi_{\text {sem }} \phi . \quad(\Leftarrow)$ Suppose $\mathcal{S} \not \mathcal{W}_{\text {sem }} \phi$. Thus, there is a $\mathcal{E} \in \operatorname{Sem}\left(\mathcal{A F}_{\vdash}(\mathcal{S})\right)$ for which there is no $a \in \mathcal{E}$ with conclusion $\phi$. By Lemma 7, there is a $\mathcal{E}^{\prime} \in \operatorname{Sem}\left(\mathcal{A} \mathcal{F}_{\vdash}\left(\mathcal{S} \cup \mathcal{S}^{\prime}\right)\right)$ for which $\mathcal{E}^{\prime} \cap \operatorname{Arg}_{\vdash}(\mathcal{S})=\mathcal{E}$. Assume for a contradiction that there is an argument $a \in \mathcal{E}^{\prime}$ with $\operatorname{Conc}(a)=\phi$. By the Pre-Relevance of $\vdash$, there is an $a^{\prime}=\left(\Gamma^{\prime}, \phi\right) \in \operatorname{Arg}_{\vdash}(\mathcal{S} \cap \operatorname{Supp}(a))$. By Fact $4, a^{\prime} \in \mathcal{E}$ which contradicts our main supposition. Thus, $\mathcal{S} \cup \mathcal{S}^{\prime} \not \psi_{\text {sem }} \phi$.

\subsection{Semantic Relevance}

Theorem 2 is a direct consequence of Theorem 4 below.

Remark 9. The grounded extension can be characterized inductively: $\operatorname{Grd}\left(\mathcal{A F}_{\vdash}(\mathcal{S})\right)=\bigcup_{\alpha \geq 0} \operatorname{Grd}_{\alpha}\left(\mathcal{A} \mathcal{F}_{\vdash}(\mathcal{S})\right)$ where $\operatorname{Grd}_{0}\left(\mathcal{A F}_{\vdash}(\mathcal{S})\right)=\operatorname{Defended}\left(\emptyset, \mathcal{A F}_{\vdash}(\mathcal{S})\right)$, $\operatorname{Grd}_{\alpha+1}\left(\mathcal{A F}_{\vdash}(\mathcal{S})\right)=\operatorname{Defended}\left(\operatorname{Grd}_{\alpha}\left(\mathcal{A F}_{\vdash}(\mathcal{S})\right), \mathcal{A F}_{\vdash}(\mathcal{S})\right)$ for successor ordinals $\alpha+1$, and $\operatorname{Grd}_{\beta}\left(\mathcal{A} \mathcal{F}_{\vdash}(\mathcal{S})\right)=$ $\operatorname{Defended}\left(\bigcup_{\alpha<\beta} \operatorname{Grd}_{\alpha}\left(\mathcal{A} \mathcal{F}_{\vdash}(\mathcal{S})\right), \mathcal{A F}_{\vdash}(\mathcal{S})\right)$ for limit ordinals $\beta$.

Theorem 4. Where $\vdash$ satisfies Cut and $\widehat{r}$ is pointed, if $\mathcal{A F}_{\vdash}(\mathcal{S}) \sim_{\text {grd }} \phi$ then

1. there is a $(\Phi, \phi) \in \operatorname{Grd}\left(\mathcal{A} \mathcal{F}_{\vdash}(\mathcal{S})\right)$,

2. $\operatorname{Grd}\left(\mathcal{A F}_{\vdash}(\mathcal{S})\right) \subseteq \operatorname{Grd}\left(\mathcal{A F}_{\vdash+\phi}(\mathcal{S})\right)$,

3. $\operatorname{Grd}\left(\mathcal{A F}_{\vdash+\phi}(\mathcal{S})\right) \cap \operatorname{Arg}_{\vdash}(\mathcal{S})=\operatorname{Grd}\left(\mathcal{A F}_{\vdash}(\mathcal{S})\right)$,

4. for every $a=(\Gamma, \gamma) \in \operatorname{Grd}\left(\mathcal{A F}_{\vdash+\phi}(\mathcal{S})\right) \backslash \operatorname{Arg}_{\vdash}(\mathcal{S})$, $(\Gamma \cup \Phi, \gamma) \in \operatorname{Grd}\left(\mathcal{A F}_{\vdash}(\mathcal{S})\right)$.

Proof. Ad 1. This is due to the fact that $\mathcal{A} \mathcal{F}_{\vdash}(\mathcal{S}) \sim_{\text {grd }} \phi$.

$A d 2$. We give an inductive proof and show the inductive step for a successor ordinal $\alpha+1$. Let $a \in \operatorname{Grd}_{\alpha+1}\left(\mathcal{A F} \mathcal{F}_{\vdash}(\mathcal{S})\right)$. Suppose $b=(\Gamma, \gamma) \in \operatorname{Arg}_{\vdash+\phi}(\mathcal{S})$ attacks $a$. If $b \in \operatorname{Arg}_{\vdash}(\mathcal{S})$ there is a $c \in \operatorname{Grd}_{\alpha}\left(\mathcal{A} \mathcal{F}_{\vdash}(\mathcal{S})\right)$ that attacks $b$. By the inductive hypothesis $(\mathrm{IH}), c \in \operatorname{Grd}\left(\mathcal{A F}_{F^{+\phi}}(\mathcal{S})\right)$. Otherwise, by Cut $b^{\prime}=(\Gamma \cup \Phi, \gamma) \in \operatorname{Arg}_{\vdash}(\mathcal{S})$ and $b^{\prime}$ attacks $a$. Thus, there is a $d \in \operatorname{Grd}_{\alpha}\left(\mathcal{A F}_{\vdash}(\mathcal{S})\right)$ that attacks $b^{\prime}$ in some $\beta \in \widehat{\Gamma \cup \Phi}$. Since $\widehat{\cdot}$ is pointed, $\beta \in \widehat{\Gamma} \cup \widehat{\Phi}$. Since $(\Phi, \phi) \in \operatorname{Grd}\left(\mathcal{A F}_{\vdash}(\mathcal{S})\right)$, $\beta \in \widehat{\Gamma}$ and hence $d$ attacks $b$ in $\mathcal{A F}_{\vdash+\phi}(\mathcal{S})$. By IH, $d \in$ 
$\operatorname{Grd}\left(\mathcal{A F}_{F^{+\phi}}(\mathcal{S})\right)$. Altogether this shows that $a$ is defended by $\operatorname{Grd}\left(\mathcal{A F}_{\vdash+\phi}(\mathcal{S})\right)$ and thus $a \in \operatorname{Grd}\left(\mathcal{A F}_{1+\phi}(\mathcal{S})\right)$.

Ad 3 and 4. We show both simultaneously via induction. We show the inductive base. Let $a=(\Gamma, \gamma) \in$ $\operatorname{Grd}_{0}\left(\mathcal{A F}_{\vdash+\phi}(\mathcal{S})\right)$. Suppose first that $a \in \operatorname{Arg}_{\vdash}(\mathcal{S})$. Since $\operatorname{Arg}_{\vdash}(\mathcal{S}) \subseteq \operatorname{Arg}_{\vdash+\phi}(\mathcal{S})$, there are no attackers of $a$ in $\operatorname{Arg}_{\vdash}(\mathcal{S})$ and hence $a \in \operatorname{Grd}_{0}\left(\mathcal{A} \mathcal{F}_{\vdash}(\mathcal{S})\right)$. Suppose now that $a \notin \operatorname{Arg}_{\vdash}(\mathcal{S})$. By Cut, $a^{\prime}=(\Gamma \cup \Phi, \gamma) \in \operatorname{Arg}_{\vdash}(\mathcal{S})$. Suppose some $b \in \operatorname{Arg}_{\vdash}(\mathcal{S})$ attacks $a^{\prime}$ in some $\beta \in \widehat{\Gamma \cup \Phi}$. By the pointedness of $\widehat{\imath}, \beta \in \widehat{\Gamma} \cup \widehat{\Phi}$. Note that $\beta \notin \widehat{\Gamma}$ since otherwise $b$ attacks $a$ but $a$ has no attackers. Thus, $\beta \in \widehat{\Phi}$. Hence, $b$ attacks $(\Phi, \phi)$ and is thus attacked by $\operatorname{Grd}\left(\mathcal{A F}_{\vdash}(\mathcal{S})\right)$.

Our previous result does not generalize to preferred semantics or to $\vdash$ that do not satisfy Cut. We give two examples.

Example 13 ([Makinson, 2003]). Consider an ASPIC framework with defeasible rules $\mathcal{D}=\left\{n_{0}: \top \Rightarrow p ; n_{1}: p \vee q \Rightarrow\right.$ $\neg p$ \}, facts $\mathcal{P}=\emptyset$, the strict rules induced by classical logic (see Ex. 12), and $\bar{\phi}=\psi$ if $\phi=\neg \psi$ and $\bar{\phi}=\neg \phi$ else. Consider the ASPIC-arguments $\mathrm{a}_{0}=\top \Rightarrow p ; \mathrm{a}=\mathrm{a}_{0} \rightarrow(p \vee q)$ and $\mathrm{b}=\mathrm{a} \Rightarrow \neg p$. With (†aspic) we have the arguments $a_{0}=\left(\left\{n_{0}, \top \Rightarrow p, p\right\}, p\right), a=\left(\left\{n_{0}, \top \Rightarrow p, p\right\}, p \vee q\right)$ and $b=\left(\left\{n_{0}, n_{1}, \top \Rightarrow p, p, p \vee q \Rightarrow \neg p, \neg p\right\}, \neg p\right)$ in $\mathcal{A} \mathcal{F}_{\vdash}(\mathcal{S})$ where $\mathcal{S}=\left\{n_{0}, \top \Rightarrow p, n_{1}, p \vee q \Rightarrow \neg p, p, \neg p\right\}$. Note that $b$ attacks $a_{0}, a$ and $b$ while $a_{0}$ attacks $b$. Thus, the only preferred extension contains both $a_{0}$ and $a$ which means that $\mathcal{S} \sim_{\text {Prf }} p$ and $\mathcal{S} \sim \sim_{\text {Prf }} p \vee q$. Once we move to $\vdash^{+(p \vee q)}$ we also have the argument $c=\left(\left\{n_{1}, p \vee q \Rightarrow \neg p, \neg p\right\}, \neg p\right)$ attacking $a_{0}$. It is easy to see that now $\mathcal{S} \not \psi_{\operatorname{Prf}}^{+(p \vee q)} p$.

Example 14. We now consider the same example but with $\vdash_{\star}=\{(\Gamma, \phi) \in \vdash \mid \Gamma$ is CL-consistent $\}$ and grounded extension. Unlike Ex. 13, $b$ is not anymore in $\mathcal{A F}_{\vdash_{\star}}(\mathcal{S})$. Thus, $\mathcal{S} \sim_{\mathrm{Grd}}^{\mathcal{A} \mathcal{F}_{\vdash}} p$ and $\mathcal{S} \sim_{\mathrm{Grd}}^{\mathcal{A} \mathcal{F}_{\vdash \star}} p \vee q$. Once we move to $\vdash_{\star}^{+(p \vee q)}$, $c=\left(\left\{n_{1}, p \vee q \Rightarrow \neg p, \neg p\right\}, \neg p\right)$ again attacks $a_{0}$ and $a$ and thus, $\mathcal{S} \mathcal{L}_{\mathrm{Grd}}^{\mathcal{A F}_{\vdash \star}^{+(p \vee q)}} p$. Note that $\vdash_{\star}$ does not satisfy Cut.

\section{Conclusion}

In this paper we investigated the robustness of systems of structured argumentation under the addition of irrelevant information. To this end we introduced a simple and easily accessible account of structured argumentation in which meta-theoretic properties can be studied conveniently while systems from the literature can be represented. We studied the properties Non-Interference, Crash Resistance, and Cumulativity. In future work we plan to incorporate priorities (e.g., [Arieli et al., 2018; Cyras and Toni, 2016]) and to extend our study to other meta-theoretic properties, such as other properties of non-monotonic inference and rationality postulates.

\section{Acknowledgments}

The authors are supported by the Alexander von Humboldt Foundation and the German Ministry for Education and Research. The first author is also supported by the Israel Science Foundation (grant 817/15).

\section{References}

[Arieli and Straßer, 2015] Ofer Arieli and Christian Straßer. Sequent-based logical argumentation. Arg\&Comp, 6(1):73-99, 2015.

[Arieli et al., 2018] Ofer Arieli, AnneMarie Borg, and Christian Straßer. Prioritized sequent-based argumentation. In Proceedings AAMAS 2018, 2018. Forthcoming.

[Avron, 2014] Arnon Avron. What is relevance logic? Annals of Pure and Applied Logic, 165(1):26-48, 2014.

[Avron, 2016] Arnon Avron. RM and its nice properties. In J. Michael Dunn on Information Based Logics, pages 1543. Springer, 2016.

[Besnard and Hunter, 2014] Philippe Besnard and Anthony Hunter. Constructing argument graphs with deductive arguments: a tutorial. Arg\&Comp, 5(1):5-30, 2014.

[Bondarenko et al., 1997] Andrei Bondarenko, Phan Minh Dung, Robert A. Kowalski, and Francesca Toni. An abstract, argumentation-theoretic approach to default reasoning. ArtInt, 93(1):63 - 101, 1997.

[Caminada et al., 2011] Martin Caminada, Walter Carnielli, and Paul Dunne. Semi-stable semantics. Journal of Logic and Computation, 22(5):1207-1254, 2011.

[Caminada et al., 2014] Martin Caminada, Sanjay Modgil, and Nir Oren. Preferences and unrestricted rebut. In Proc. COMMA'14, pages 209-220, 2014.

[Cyras and Toni, 2016] Kristijonas Cyras and Francesca Toni. ABA+: assumption-based argumentation with preferences. In Proc. KR'2016, pages 553-556, 2016.

[Dung, 1995] Phan Minh Dung. On the acceptability of arguments and its fundamental role in nonmonotonic reasoning, logic programming and n-person games. ArtInt, 77(2):321-357, 1995.

[Grooters and Prakken, 2016] Diana Grooters and Henry Prakken. Two aspects of relevance in structured argumentation: Minimality and paraconsistency. Journal of Artificial Inteligence Research, 56:197-245, 2016.

[Heyninck and Straßer, 2016] Jesse Heyninck and Christian Straßer. Relations between assumption-based approaches in nonmonotonic logic and formal argumentation. In Proc. NMR'16, 2016.

[Heyninck and Straßer, 2017] Jesse Heyninck and Christian Straßer. Revisiting unrestricted rebut and preferences in structured argumentation. In Proc. IJCAI'17, pages 10881092, 2017.

[Makinson, 2003] David Makinson. Bridges between classical and nonmonotonic logic. Logic Journal of IGPL, 11(1):69-96, 2003.

[Prakken, 2010] Henry Prakken. An abstract framework for argumentation with structured arguments. Arg\&Comp, 1(2):93-124, 2010.

[Wu and Podlaszewski, 2014] Yining Wu and Mikołaj Podlaszewski. Implementing crash-resistance and noninterference in logic-based argumentation. Journal of Logic and Computation, 25(2):303-333, 2014. 